\title{
Mal de Pott: um diagnóstico improvável
}

Maria Inês Silva, ${ }^{1}$ Joana Pinto, ${ }^{1}$ Manuel Viana ${ }^{1}$

\section{RESUMO}

Introdução: As manifestações osteoarticulares da tuberculose são a terceira apresentação mais frequente da tuberculose extrapulmonar, sendo o esqueleto axial o local mais afetado, o que se designa por espondilodiscite tuberculosa ou Mal de Pott. Apresenta-se um caso clínico que pretende alertar para este diagnóstico, pouco frequente nos cuidados de saúde primários, cuja clínica inespecífica e insidiosa pode dificultar e atrasar o diagnóstico e o tratamento.

Descrição do caso: Homem, 82 anos, nacionalidade portuguesa, com antecedentes pessoais de hipertensão arterial medicada e controlada. Sem outros antecedentes pessoais de relevo, nomeadamente infeção pelo vírus da imunodeficiência humana (VIH) ou imunossupressão. Desconhece antecedentes pessoais de tuberculose ou contactos de risco. Recorre à consulta aberta da sua Unidade de Saúde Familiar por dor na região dorsal e no hemitórax esquerdo com cerca de quatro meses de evolução, com uma intensidade de 6 em 10, sem irradiação, com alívio parcial com medicação analgésica e anti-inflamatória e agravamento com os movimentos. Sem história de traumatismo. Nega sintomatologia respiratória, neurológica e outros sintomas constitucionais gerais. O exame objetivo não evidenciou alterações à observação da coluna dorsal, nomeadamente sinais inflamatórios locais ou deformação visível da coluna, nem dor à palpação e nem alterações da sensibilidade ou força muscular. Realizou radiografia da coluna dorsal e radiografia do tórax que não revelaram alterações significativas. Dada a evolução temporal optou-se por realizar tomografia computorizada da coluna dorsal que revelou alterações fortemente sugestivas de um processo de espondilodiscite. O doente foi referenciado para o serviço de urgência hospitalar, onde foi proposto internamento para estudo e orientação, do qual se realça biópsia óssea positiva para polymerase chain reaction (PCR) de bacilo de Koch. Foi assumido o diagnóstico de Mal de Pott, tendo o doente iniciado tratamento com anti-bacilares.

Comentário: O Mal de Pott tem como sintoma mais comum a dor localizada, com agravamento progressivo ao longo de semanas ou meses, ou seja, uma clínica pouco específica e insidiosa, o que contribui para o atraso no diagnóstico. Podem existir sintomas constitucionais, como perda de peso ou febre, embora estes estejam presentes em menos de metade dos casos. Os métodos de imagem são importantes para o diagnóstico, nomeadamente a tomografia computorizada e a ressonância magnética, sendo a radiografia da coluna pouco sensível, particularmente na fase inicial. Após o diagnóstico, o tratamento deve ser instituído de forma célere com o fim de melhorar o prognóstico. O médico de família tem um papel importante quer na suspeita diagnóstica desta patologia, permitindo o diagnóstico e tratamento atempados, quer no seu seguimento, apoiando a adesão ao tratamento e a gestão das complicações da doença.

Palavras-chave: Infeções por micobactérias; Tuberculose; Tuberculose osteoarticular; Tuberculose axial.

\section{INTRODUÇÃO}

$\wedge$ tuberculose continua a ser uma causa major de mortalidade a nível mundial. ${ }^{1} \mathrm{O}$ bacilo responsável por esta patologia, Mycobacterium tuberculosis, afeta tipicamente os pulmões, mas poderá afetar outros locais, designando-se nesse caso por tuberculose extrapulmonar. As manifestações osteoarticulares são a terceira apresentação mais frequente deste tipo de tuberculose, correspondendo de

1. USF São João do Porto. Porto, Portugal.
1 a $5 \%$ do total de casos. ${ }^{2} \mathrm{O}$ esqueleto axial é o local mais afetado e esta patologia designa-se por espondilodiscite tuberculosa ou Mal de Pott, que aparece mais frequentemente ao nível da região dorsal. ${ }^{3-4}$

Com a diminuição da incidência da tuberculose pulmonar, a proporção de casos de tuberculose extrapulmonar parece estar a aumentar. ${ }^{5} \mathrm{Na}$ Europa, a proporção de casos de tuberculose extrapulmonar aumentou de 16,4\% em 2002 para 22,4\% em 2011. ${ }^{6}$ Similarmente, nos Estados Unidos houve um aumento: de 15,7\% em 1993 para $21 \%$ em $2006 .{ }^{2}$ 
Apesar de todos os avanços tecnológicos, a espondilodiscite tuberculosa continua a ser um grande desafio diagnóstico, já que requer elevada suspeição clínica. Mesmo sendo uma entidade com baixa mortalidade, está associada a morbilidade significativa, sendo que um diagnóstico tardio pode levar a deformidades esqueléticas importantes e a complicações neurológicas irreversíveis. ${ }^{7}$

O presente relato de caso pretende alertar para este diagnóstico, pouco frequente nos cuidados de saúde primários, cuja clínica inespecífica e insidiosa pode dificultar e atrasar o diagnóstico e o tratamento, levando a consequente morbilidade significativa para o doente. Pretende-se ainda discutir o papel do médico de família no diagnóstico e no seguimento desta patologia.

\section{DESCRIÇÃO DO CASO}

Homem de 82 anos, de nacionalidade portuguesa, reformado, trabalhou inicialmente como militar e posteriormente na Cruz Vermelha e como barbeiro, autónomo nas atividades de vida diária. Inserido numa família nuclear na fase VIII do Ciclo Familiar de Duvall. Vive numa habitação com boas condições higieno-sanitárias. Apresenta como antecedentes pessoais hipertensão arterial, sem atingimento de órgão-alvo, medicado com losartan 100mg e hidroclorotiazida $25 \mathrm{mg}$, um comprimido de manhã. Antecedentes de cirurgia à coluna lombar há cerca de 30 anos. Sem outros antecedentes pessoais de relevo, nomeadamente infeção pelo vírus da imunodeficiência humana (VIH) ou imunossupressão. Desconhece antecedentes pessoais de tuberculose ou contacto com pessoas com suspeita ou diagnóstico de tuberculose. Não existem registos de ter realizado vacina BCG. Doente com ingestão de cerca de $26 g$ de álcool por dia. Sem hábitos tabágicos ou consumo de drogas injetáveis.

Recorre à consulta aberta da sua Unidade de Saúde Familiar por dor na região dorsal e no hemitórax esquerdo com cerca de quatro meses de evolução. O doente relaciona esta dor com a realização de trabalhos de agricultura que envolvem a necessidade de carregar cargas pesadas, caracterizando a dor como tendo uma intensidade de 6 em 10, sem irradiação. Nega história de traumatismo. Nega outra sintomatologia associada, nomeadamente febre, tosse ou dispneia, sudorese noturna ou perda ponderal inexplicada. O doente tinha

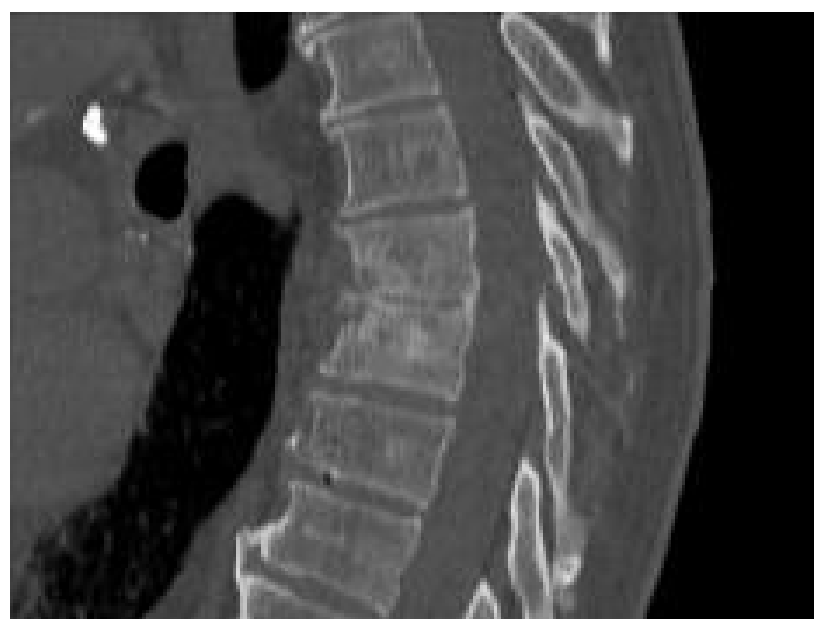

Figura 1. Tomografia computorizada da coluna dorsal com alterações sugestivas de espondilodiscite em D6-D7.

sido observado anteriormente, em contexto de consulta aberta na mesma unidade e no serviço de urgência hospitalar, tendo sido medicado com analgésicos e anti-inflamatórios não esteroides diversos, com alívio parcial da dor. Ao exame objetivo não apresentava alterações de relevo: sem alterações à observação da coluna dorsal, nomeadamente sinais inflamatórios locais ou deformação visível da coluna, sem dor à palpação da coluna e sem alterações da sensibilidade ou força muscular. Para exclusão de possíveis diagnósticos diferenciais, nomeadamente fratura da coluna, neoplasia ou infeção, realizou radiografia da coluna dorsal e radiografia do tórax que não revelaram alterações significativas. No entanto, dada a evolução temporal e a ineficácia terapêutica, optou-se por realizar tomografia computorizada da coluna dorsal que revelou "em D6-D7, diminuição do espaço discal com forte heterogeneidade de ambos os corpos vertebrais, com destruição óssea associada, aspetos fortemente sugestivos de um processo de espondilodiscite" (Figura 1).

Neste contexto, foi encaminhado para o serviço de urgência hospitalar, onde foi realizado estudo analítico (sem leucocitose, velocidade de sedimentação de $24 \mathrm{~mm}$ e proteína C reativa de $9,09 \mathrm{mg} / \mathrm{L}$ ) e hemoculturas, que foram estéreis. Foi proposto internamento para estudo e orientação, no qual se realça a realização de biópsia óssea que se revelou positiva para polymerase chain reaction de bacilo de Koch, com bacteriológico estéril. Não se encontraram achados sugestivos de 
tuberculose pulmonar ativa e a pesquisa do VIH foi negativa. Assim, foi assumido o diagnóstico de Mal de Pott, tendo o doente iniciado tratamento com anti-bacilares, com melhoria da dor.

\section{COMENTÁRIO}

O sintoma mais comum do Mal de Pott é a dor localizada que aumenta em intensidade ao longo de semanas a meses, podendo associar-se a rigidez muscular. Os sintomas neurológicos são os segundos mais frequentes. ${ }^{8}$ Em menos de metade dos casos poderão estar também presentes sintomas constitucionais, como a perda de peso ou febre. ${ }^{8}$ Existem alguns fatores de risco identificados para o desenvolvimento desta patologia, como a infeção por VIH, diabetes mellitus, doença renal crónica, uso crónico de corticosteroides, uso de drogas endovenosas ou consumo crónico de álcool. ${ }^{8}$ Uma importante questão prende-se com o facto das manifestações clínicas serem pouco específicas e de aparecimento insidioso, podendo condicionar um atraso no diagnóstico e, consequentemente, um pior prognóstico. ${ }^{8}$

O diagnóstico de suspeição é feito com base na apresentação clínica e nos achados radiológicos. A radiografia da coluna é, muitas vezes, o primeiro exame complementar a ser realizado, mas pode não apresentar alterações numa fase precoce da doença. No entanto, quando a suspeita diagnóstica surge numa fase mais avançada da doença podem já estar presentes alterações, nomeadamente destruição dos corpos vertebrais (predominantemente anterior) e dos discos adjacentes. Outros métodos de imagem, nomeadamente a tomografia computorizada e a ressonância magnética, são importantes para caracterizar a lesão, orientar a realização de biópsia, avaliar a resposta ao tratamento e possíveis complicações. ${ }^{9-10}$ Para confirmação diagnóstica é fundamental a identificação do mycobacterium tuberculosis. ${ }^{8}$

O tratamento é feito com antibacilares, podendo em determinadas situações haver necessidade de tratamento cirúrgico. O objetivo do tratamento é tratar a infeção, mas também limitar o aparecimento de complicações. O tratamento com antibacilares deve ser realizado durante seis a nove meses, sendo que a duração ótima do tratamento não está ainda estabelecida. ${ }^{11}$

Este caso clínico diz respeito a um diagnóstico que pode ser um desafio para os médicos face ao quadro clínico pouco específico e insidioso, muitas vezes sem outros sintomas associados. O médico de família tem um papel importante na suspeita diagnóstica desta patologia, permitindo o diagnóstico e tratamento atempados, o que é fundamental para um melhor prognóstico. O médico de família tem ainda um papel crucial no seguimento destes doentes, nomeadamente no que diz respeito à adesão ao tratamento prolongado e à gestão das complicações da doença, como o controlo da dor e a gestão das alterações de mobilidade, que podem condicionar limitação nas atividades de vida diária e maior risco de quedas. De salientar também que a infeção por mycobacterium tuberculosis é uma doença de notificação obrigatória e o médico de família pode ter um papel importante na articulação com a saúde pública e na gestão de contactos do doente.

\section{REFERÊNCIAS BIBLIOGRÁFICAS}

1. World Health Organization. Global tuberculosis report 2019: executive summary [Internet]. Geneva: WHO; 2019. Available from: https://www.who.int/tb/publications/global_report/GraphicExecutiveSummary.pdf?ua $=1$

2. Peto HM, Pratt RH, Harrington TA, LoBue PA, Armstrong LR. Epidemiology of extrapulmonary tuberculosis in the United States, 1993-2006. Clin Infect Dis. 2009;49(9):1350-7.

3. López-Sánchez MC, Calvo-Arrojo G,Vázquez-Rodríguez TR. Tuberculous spondylodiskitis with lumbar tumor. Reumatol Clin. 2012;8(5):292-3.

4. García-Lechuz JM, Julve R, Alcalá L, Ruíz-Serrano MJ, Muñoz P. Espondilodiscitis tuberculosa o enfermedad de Pott: experiencia en un hospital general [Tuberculous spondylodiskitis (Pott's disease): experience in a general hospital]. Enferm Infecc Microbiol Clin. 2002;20(1):5-9. Spanish

5. Pigrau-Serrallach C, Rodríguez-Pardo D. Bone and joint tuberculosis. Eur Spine J. 2013;22 Suppl 4:556-66.

6. Sandgren A, Hollo V, van der Werf MJ. Extrapulmonary tuberculosis in the European Union and European Economic Area, 2002 to 2011. Euro Surveill. 2013;18(12):20431.

7. Pellisé F. Tuberculosis and Pott's disease, still very relevant health problems. Eur Spine J. 2013;22 Suppl 4:527-8.

8. Trecarichi EM, Di Meco E, Mazzotta V, Fantoni M. Tuberculous spondylodiscitis: epidemiology, clinical features, treatment, and outcome. Eur Rev Med Pharmacol Sci. 2012;16 Suppl 2:58-72.

9. Colmenero JD, Jiménez-Mejías ME, Reguera JM, Palomino-Nicás J, RuízMesa JD, Márquez-Rivas J, et al. Tuberculous vertebral osteomyelitis in the new millennium: still a diagnostic and therapeutic challenge. Eur J Clin Microbiol Infect Dis. 2004;23(6):477-83.

10. Rivas-Garcia A, Sarria-Estrada S, Torrents-Odin C, Casas-Gomila L, Franquet E. Imaging findings of Pott's disease. Eur Spine J. 2013;22 Suppl 4:567-78.

11. Nahid P, Dorman SE, Alipanah N, Barry PM, Brozek JL, Cattamanchi A, et al. Official American Thoracic Society/Centers for Disease Control and Prevention/Infectious Diseases Society of America Clinical Practi- 
ce Guidelines: treatment of drug-susceptible tuberculosis. Clin Infect Dis. 2016;63(7):e147-e195.

\section{CONFLITO DE INTERESSES}

Os autores declaram não possuir quaisquer conflitos de interesse.

\section{FINANCIAMENTO DO ESTUDO}

Os autores não receberam qualquer financiamento para o desenvolvimento do estudo.

\author{
ENDEREÇO PARA CORRESPONDÊNCIA \\ Maria Inês Silva \\ E-mail: psilva.ines@gmail.com \\ https://orcid.org/0000-0001-6464-6677
}

Recebido em 09-04-2020

Aceite para publicação em 23-10-2020

\section{ABSTRACT}

\section{PERCEPTION OF DEPRESSION AND ANXIETY BY FAMILY PHYSICIANS ACCORDING TO PATIENT GENDER}

Introduction: Osteoarticular manifestations of tuberculosis are the third most frequent presentation of extrapulmonary tuberculosis, with the axial skeleton being the most affected site, which is called tuberculous spondylodiscitis or Pott's disease. We present a clinical case that aims to alert to this diagnosis, uncommon in primary health care, whose nonspecific and insidious clinic can hinder and delay diagnosis and treatment.

Case description: 82-year-old Portuguese man, with a personal history of Arterial Hypertension. No other relevant personal history, namely infection by the human immunodeficiency virus (HIV) or immunosuppression. He has no story of tuberculosis or risk contacts. He goes to his Health Unit with pain in the dorsal region and in the left hemithorax with about four months of evolution, with an intensity of 6 in 10, without irradiation, with partial relief with analgesic and anti-inflammatory medication, and worsening with movements. No history of trauma. He denies respiratory, neurological, and other general constitutional symptoms. On physical exam, there were no changes to the observation of the dorsal spine, namely local inflammatory signs or visible deformation of the spine, no pain on palpation, and no changes in muscle sensitivity or strength. Dorsal spine and chest X-rays were performed, and both were considered normal. Given the temporal evolution, it was decided to perform computed tomography of the dorsal spine, which revealed changes that were strongly suggestive of a spondylodiscitis process. The patient was referred to the hospital emergency service, where he was admitted for study and orientation, having a positive bone biopsy for Koch Bacillus. The diagnosis of Pott's disease was assumed, and the patient started treatment with tuberculostatic agents.

Comment: The most common symptom of Pott's disease is localized pain, with progressive evolution over weeks or months, that is, a non-specific and insidious clinic, which contributes to the delay in diagnosis. Constitutional symptoms may exist, such as weight loss or fever, although these are present in less than half of the cases. Image methods, such as computed tomography and magnetic resonance Imaging, are important for diagnosis. Spine radiography is insensitive, particularly in the initial phase. After diagnosis, the treatment should be instituted quickly to improve the prognosis. The family doctor has an important role in diagnosis and in follow-up, supporting adherence to treatment and management of disease complications.

Keywords: Mycobacterium infections; Tuberculosis; Osteoarticular tuberculosis; Spinal tuberculosis. 